Monatsschrift f. Geburtshülfe u. Gynäkologie 1921;54:136-142

\title{
III. Literatur-Verzeichnis
}

\section{Geburtshilfe.}

Schatz, Friedrich †, Nekrolog Prochownick. Arch. f. Gyn. Bd. 113. 3 f. V. Anderodias, Grippe und Schwangerschaft. Rev. mens. de Gynéc. et. obst.

19. Juni. Aubert, Fall von allgemeiner eitriger Peritonitis nach kriminellen Abort.

Laparatomie, Enterostomie. Heilung. Rev. mens. de gynéc. et

d'obst, 19. Mai. Bacon, Ch., Verkürzung der Nabelschnur. Siirg. gynéc. et obst. XXX. S. 6.

Bailey, Geburtsbeschädigung des Schädels und seines Inhalts. Americ.

J. obst, gynec. I. H. 1. S. 52. Betz, Intrauterine Cholevalspülungen bei gynäkologischen

Operationen.

Munch, med. Woch. 20. No. 13. Bosset, Schmerzlinderung unter der Geburt. Schweiz.

Rundschavi f. Med. 19.

No. 22. Beuítne $\Gamma$ und Vullietz, Grippe und Gestation. Schweiz. med. Woch. 20.

No. 4.

Literatur-Verzeichnis.

137

Borurika, Zwei interessante Fälle von kriminellen Abortus. Cas. lék. cerk.

20. No. 15. Buchacker, Ein Beitrag zur Frage der Geschlechtsbildung. Zbl. f. Gyn.

20. XL S. 1345. Bucura, Mutterschutz. Wien med. Woch. 19. No. 33 u. 34. Bullowa und Breman , Intrauterine Intestinalobstruktion durch ein-

gedicktes und eingepreßtes Meconium. J. Am. u. m. Ass. 19. No. 25. Burger, Die katholische

Hebamme im Dienst der Seelsorge. Freiburg, B.

Herder. 20. Cathala, Die Inzisionen der Portio vaginalis des Collum uteri während der rechtzeitigen Geburt. Gynéc. et obst. 20. I. 2. . Catinat, Die vaginale Totalexstirpation des Uterus bei puerperaler Inf ektion.

Presse med. 20. No. 4. S. 33. Couínaud, Umwandlung einer Seheitel- in eine Gesichtslage während der

Zangenextraktion bei kyphotischem Becken. Gynéc. et obstét. 1920.

No. 2. S. 147. Creadrick, Häufigkeit und Bedeutung der Omphalitis. Surg. Gynec. et obst. XXX. No. 2. Cull, Spina bifida und diesbezügliche Störungen bei einem menschlichen 17 mm langen Embryo. Bull. Johns Hosp. XXX. S. 340. Dalyell, Von den Gewichtskurven einiger Fälle von Säuglingsskorbut in

Wien. Wien. med. Woch. 1920. No. 9. Dietrich, H. A., Einführung in Geburtshilfe und Gynäkologie. Munch.

u. Wiesbaden, J. F. Bergmann. 1920. Döderiein Handbuch der Geburtshilfe. 3. Bd. Baisch, Pathologie der

Geburt, - F. Weber, Die mehrfache Schwangerschaft. - - Die Entzündungen der Brustdrüse (Mastitis). @- - P. Zweifel, Das Kindbettfieber. - Siegel, Genitalblutungen bei Wöchnerinnen. - Stoeckel, Die Harnorgane in der Schwangerschaft, während der Geburt und im 
Wochenbett. - Ibrahim, Krankheiten der Neugeborenen. üörner, Scharlach bei Neugeborenen und Säuglingen. Dtsch. med. Woch. 20.

No. 27. Dorsath, Ruptur des Rektum während der Geburt. Surg. gynéc. et obst.

XXX. No. 2. Ëkler, Gravidität in einer Nebentube. Arch. f. Gyn. Bd. 113. H. 3. S. 6083. Essen-

Möller, Ein Mittel vor der Geburt eine Drillingsschwangerschaft

zu diagnostizieren. Gynéc. et obstét. No. 2. 1920. S. 145. Everke, K., Zur Behandlung des

Schwangerschaftserbrechens und der

Eklampsie mit Ovoglandol nach Hofbauer. Zbl. f. Gyn. 16. X.

S. 1278. Frankl, Die NaCl-Fällung der Plazenta (Bemerkungen zu der gleich-

namigen Arbeit von Traugott. Dtsch. Woch. No. 21. 20. Munch.

med. Woch. 19. XI. S. 1357. - Traugott, Erwiderung auf die Bemerkungen von D. 0. Frankl. S. 1357. Freund, Herm., Abriß der geburtshilflichen Operationslehre. Reichs-

Medizinalkalender 1921. Thieme, Leipzig. S. 239. Fuchs, Vagitus uterinus. Zbl. f. Gyn. 13. XL 20. S. 1310. Gautico (Prof. Dr. jur.), Über therapeutischen Abort. Schweiz. med. Woch.

20. No. 3.

138

Literatur-Verzeichnis.

Geymüller, Einfluß der Influenza auf Schwangerschaft iind Wochenbett.

Korresp.-B1. f. Schw. Ärzte 19. No. 32. McGlim, Sectio eaesaГra extraperitonealis. Amer. J. obstets gyn. I. H. 1.

S. 45. Grosse, Spontangeburten nach Kaiserschnitt bei derselben Frau. Gynec.

et obst. 20. No. 2. S. 81. Hauch und E. Ruge, Das Ergebnis der Behandlung der Neugeborenen.

Gynéc. et obst. No. 2. S. 84. Hainiss, EinigeWorte zumArtikel Szancau Pötes. Orvosi Hetilap 19.

No. 31. Haschting, Über den Einfluß des Kriegs auf Präservativverkehr und

Fruchtabtreibung und seine körperlichen Folgen. Frauenarzt 19.

J. 9, 10. Hart, Berry, Über die Ursaehen des Puerpeialfieber in den antiseptischen

Ära. Edinb. m. J. XXVI. No. 1. Heim, Diätetik der stillenden Mutter. Budapesti Orvosi Upsacj

19. No. 5. Heinrichsdorff, Sopor und Eklampsie. Arch. f. Gyn. Bd. 113. H. 3. S. 596. Hiess,

Bemerkungen über die normale Lösung der Plazenta. Wien. klin.

Woch. 14. X. S. 927. Hirsch, M., Gedanken und Vorschläge zur Neuorclnung des

geburtshilflich-

gynäkologischen Unterriehts. Arch, f. Frauenkunde und Eugenotik V].

H. 3/4. Hornung, Beitrag zu den Beziehungen zwischen Syphilis und Schwangerschaft. Zbl. f.

Gyn. 23. X. S. 1222. HampstoПe, Schwangerschaft in einem rudumentären Uterushorn. Sui $\cdot g$.

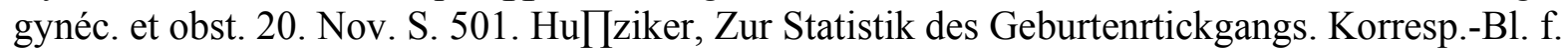
Schweizer

Ärzte. 19. No. 46. Hüssy, Die moderne Erklärung der Schwangerschaftstoxikosen. Schweiz. med. Woch. 13. IX. 20. S. 857. Jeard, Der Beweis des intrauterinen Lebens. Neue Methoden der Lungen-

probe. Gaz. d. hopit. 19. No. 61. Jackson, Akute Darmlähmung infolge von Schwangerschaft in einem

Uterus bicorais. Brit. med. J. No. 3084 Jaeeger, Vergleichende Ti rexperimentelle und klinische Versuche mit

. Sekaleersatz. Zbl. f. Gyn. 23. X. S. 1211. Janácek, Schwangerschaftstatonie mit beiderseitiger Cataracta. Cas 
Okarina cesk. 20. No. 10. Jurcev, Beitrag zur puerperalen Uterusinversion. Wien, klin. Woch. 19.

No. 37. Jaschke, v., Die Stellung der Nephropathia gravidarum im System der

Nierenerkrankungen. Zbl. f. Gyn. 6. XI. S. 1273. Jellet, Modifikationen der Hebesteotonie. Surg. gynéc. et obst. XXX.

No. 6. Jung, Der therapetitische Abort im heutigen Strafrecht der Kantone.

Schweiz. med. Woch. 20. No. 3. Kafka, Ein Symptom zur klinischen Diagnose der fötalen Hydros universalis

in utero. Zbl. f. Gyn., 20. XI. S. 1343. Kalliwoda, Mißerfolge der Tubensterilisationen, ihrer Genese und Prophylaxf.

Zugleich ein Beitrag zur Kasuistik der Schwangerschaften nach

Literatur-Vßrzeíchuis. 139

Inteipositio uteri vescio-vaginalis. Arch. f. Gyn. Bd. 113. H. 3.

S. 565. Stein, A., Gr $\theta$ burtshilflich $\theta$ Tagesfragen. 1. Über die Anwendung minimaler

Dosen $\backslash$ ion Pituitrin zur Einleitung der Geburt am Ende der Schwanger-

schaft. - 2. Über die Stickstoffoxydul-Sauerstoffnaikose als Ersatz

des Dämmerschlafes zur Herbeiführung eiuer völlig schmerzlosen

Geburt, - Zbl. f. Gyn. 9. X. S. 1152. Strachmann, Zur mikroskopischen Untersuchung von

Lungen Neugeborener.

Dtsch. med. Woch. 20. Nr. 14 u. 15. Strakosch, Zur Abortbehandlung. Zbl. f. Gyn. 9. X. S. 1155

Straßmann, G., Einiges zur sanitätspolizeilichen Bedeutung des Kindbett-

fiebers. Ztschr. f. Mediz.-Beamte. 20. Nr. 2. Streeter, Bildung eineiiger Zwillinge. Bull, Johns Hopkins Hosp. XXX.

S. 342. Sundo, Ein Fall von Ileus im Wochenbett mit einem abnormen Verhalten

des Dünndarm. Norsk, mag. f. laegeridenk. 18. S. 303. - Szana u. Totis, Verdauungsstöi'ungen

der mangelhaft ernährten Säuglinge.

Orvosi Hetilap. 19. Nr. 26. Szana, Antwort auf, diesen Artikel. Orvosi Hetilap. Nr. 31. Torggler, Geburtshilflich-gynäkologische Beobachttingen bei Grippe-

erkrankungen. Frauenarzt 19. H. 7 u. 8. Tóth, Über den künstlichen Abort. Orvos képsés. 19. Nr. 1-3. Truska, Streptothrix in den Genitalien schwangerer Frauen. Casopis

lék. ceskych. 19. Nr. 45-46. Umber, Das Coma diabetieum bei Schwangeren. Dtsch. med. Woch. 20.

Nr. 28. Vignes, Intrauterine Therapie bei uteriner Infektion post partum. Gynéc.

et obstét, II.' Nr. 3. S. 161. Wächter, Beitrag zur Kasuistik und Therapie der Placenta praevia.

Schweiz.

m. W. 15. VII. 20. S. 618. Ward, Herzmassage bei Asphyxin der Neugeborenen. Brit. in. J. 20.

Nr. 3086. Werner, Drohende Uterusruptur bei Partus praematurus, gleichzeitig ein

Beitrag zur Dekapitation. Zbl. f. Gyn. 23. X. S. 1216. Zelnick, Über die Behandlung des

fiebernden Abortes. Wien. kl. W. 20.

Nr. 27.

Gynäkologie.

Anschütz, Über Hautanaesthesie bei Nabel- und Bauchbrüchen. Beitr.

z. kl. Chir. CXV. S. 2. Aerni, Die Thermo -Präzipitinreaktion als Diagnostikum bei Gonorrhöe.

Schweiz. Rundschau f. Med. 18. Nr. 14. Asch, Ztir Ha\%oitnaht, Zbl. f. Gyn. 13. XI. 20. S. 1314.

Bergé, Vallory, Radot u. Arnold, Cholesterincyste der Ovarien. Bull, et 
mem. de la soc. anatom. Paris 20. März. Bloch, Über die Schwierigkeiten der Diagnose der stielgedrehten Ovarial-

cysten. Gynéc. 20. Febr. Bock, Die Vulvovagitis gonorrhoica infantum, ihre Therapie und deren Erfolg. Frauenarzt 19. S. 2 u. 3.

140

Literatur-Verzeichnis.

Borée, Warnung gegen die wahllose uterine Curettage. Surj. Gynéc. a

obst. XXX. N. 6. Briau, Lacassagne u. Lagoutte, Ein Fall von doppe $1 / 8$ eitigem Hermaphroditis-

mus mit bisexuellen Geschlechtsdrüsen. Gynéc. et obst. I. Nr. 20. Brunn, M. v., Über

verschiedene Narkoseverfahren. Zbl. f. Chir. 19. H. 19. Brunn, M. v., Zur Frage der

Lumbalanästhesie. Zbl. f. Chir. 19. H. 30. Cadwallada, Solitäre Leistenliernie des Ovarium. J.

Am. m. Ass. 23. X. 20.

S. 1137. Caulk, Experimentelle Studie über die Ligierung des Ureters. Surg. gyn;

a. Obst, 1920. XXX. Nr. 4. Chambers u. Durand, Großes Fibrosarkoni des Ligulatum mit

zentraler

Ekrose. Bull, et mem. soc. anatom. Paris. 09. Dez. Chavannaz u. Nadal Mischgeschwülste des

Uterus. Gynec, 20. Jan. Chavannas, Urachuszysten. Ann. de Gyn. et d'obst. 17. Juli-August.

Chastenet de Geru, Die Gaszysten des Abdomen. Gaz. des hop. 19. Nr. 78. Chauvín u. Roux,

Kontaktmetastase eines Collumkarzinom auf emem

Uteruspolypen, Bull, et mém. de la soc. anatom. 20. März. Calmann, Ausgedehnte

Gewebsnekrose nach Amnesie. Dtsch. med. Woch.

20. Nr. 5. Condit, Gutartige Geschwülste der Labien. Surg. gynéc a, obst. Nov.

20. S. 487. Dan forth, N iereninf ektionen in der gynäkologischen Praxis. Surg. gynéc.

a. obst. 1920. Nr. 2. Descomps u. Hue, Intraligomentäres Myom. Gynéc. Feb. 20. Dickinson Ein

Programm für die amerikanische Gynäkologie. Präsidenten-

reden $1 / 8$ der amerikanischen gynäkologischen Gesellschaft. American

J. of obstet. a, gynec. I. H. 1. S. 2. Digby, Der Nutzen des doppelten Fadens bei chirurgischer

Arbeit. Surg.

gyn. obst. Oct. S. 416. Ducuiny, Symmetrische kongenitale Lipome der Ligg. rot. Gyn. et obst.

12. H. 1. Diijarien u. Topous Khan, Großes gestieltes subseröses Myom, vom Uterus

abgetrennt und festgewachsen am S. romanum. Bull, et mem. Soc.

anat. Paris. 20. Nr. 7. Ebeler, Die Beeinflussung klinakterischer Blutungen durch Radium. Med K1. 24. X. S. 1108 und 31. X. S. 1134. Ehrenfest, Prophylaxe und Behandlung der puerperalen Infektionen. Kriti-

sches Sammelreferat. Americ. J. obstet. gyn. I. 1. S. 103. Farr, Der 3. und 4. Zangenknoten. Surg. gyn. a, obst. Okt. S. 408. Finsterer, Nochmals zur Frage der Lokalanästhesie bei den großen Bauch-

operationen. Wien. med. Woch. 19. Nr. 48. Finsterer, Schlußwort zur Frage der Lokalanästhesie bei den großen Bauch-

operationen. Wien. med. Woch. 19. Nr. 50. Frank, R. T., Einfluß des Hypophysenextraktes auf den Genitaltraktus.

Journ. am. m. Ass. 19. Nr.. 23. Froriep, Die Behandlung infektiöser Scheidenerkrankungen mit Jod-

tinktur. Munch, med. Woch. 15. X. S. 1202. Fuchs, Dora, Spirochaeta pallida, Befund in der

Cervix bei primärer Lues.

Dtsch. med. Woch. 14. X. S. 1165. 
Literatur-Verzeichnis. 141

Loeser, Konstitution und latente Infektion (Mikrobismus), mit besonderer

Berücksichtigung der Scheidenflora und des Puerperalfiebers. Zbl.

f. Gyn. 30. X. S. 1254. Marion, Behandlung der Blasen-Scheidenfistein auf transvesikalem Weg. Gynec. 20. März. Maxwell, Verkalkte Tubenmole. Surg. gyn. a. obst. Oktober. S. 388. Mayo, Ch., Beziehungen des Krebses zur Verlängerung des Lebens. Surg.

gynéc. et obst. XXX. Nr. 1. Melchior, Über sekundäre Enterostomien nach Peritonitis uncl Ileusopera.

tionen. Bed. klin. Woch. 20. Nr. 3. Menge u. Opílz, Handbuch der Frauenheilkunde für Ärzte und Studierende.

Bearbeitet von Amann, Baisch, Beuttner, v. Franqué, Füth, Halban > v. Jaschke, Jung, Knauer, Menge. Opitz, Pankow, Rei†ferscheid, Schroder, Sellheim, Tandler, Walthard. 2. u. 3. Aufl. München u. Wiesbaden.

1920. J. F. Bergmann. Meredith, Fl., Funktionelle Operationsbeschwerden. Surg. gyn. a. obst. Okt. 20. S. 382. Micholitsch, Scheintumoren im Bauch. Zbl. f. Gyn. 16. X. S. 1184. Nairn, Hernia properitonealis und spontane Scheinreduktion. Arch. f. kl.

Chir. CXIII. H. 4. Newton Evans, Maligne Myome und verwandte Tumoren des Uterus.

Surg. gyn. a. obstet. XXX. Nr. 2. Ochsner, Weitere Beobachtungen über das Corpus luteurn. Surg. gynéc.

obst. Nov. 20 S. 496. Otlow, Zur Kritik der Nagel• Ve³/8chen Gynatresielehre. Zbl. f. Gyn. 13. XI.

20. S. 1305. Pamboukis, Die Autointoxikationen ovariellen Ursprungs. Gaz. des hopitans. 19. Nr. 77. Peraire, Tuberkulose des Uterus und der Adnexe, angefacht durch gewalt same Uterusdilatation. Bull, et mem. de la soc. anatom. de Paris.

“20. H. 2. Pfanner. Zum Artikel Finsterers: Nochmals zur Frage der Lokalanästhesie bei den großen Bauchoperationen. Wien. med. Woch. 19. Nr. 49. Reynolds Praktische Gesichtspunkte und gewöhnliche Fehler bei der

Behandlung der Sterilität. Journ. A. m. Ass. 19. Nr. 15. Richter, H. M., Eine neue Operation gegen Rekotrumvorfall bei Frauen.

Surg. gynéc. a. obst. 20. Nov. S. 526. Roesch, Über die Beziehungen der Sklerodermie zu den Erkrankungen der

endokrinen Drüsen. Dtsch. med. Woch. 19. Nr. 48. Schäfer, P., Resultate der Collifixatio uteri. Arch. f. Gyn. Bd. 113. H. 3.

S. 588. Schepelmann, Die Bauchdeckenplastiken mit besonderer Berücksichtigung des Hängebauches. \{Bruns Beitr.) K1. Ch. CXI, H. 2. Schloßmann, Über die Geburtenhäufigkeit und die Säuglingssterblichkeit

nach dem Kriege. Dtsch. med. Woch. 1. X. S. 1104. Seelis, Pseudomyxoma peritonei. Surg.

gynéc. a. obst. XXX. Nr. 6. Shoemaker, Behandlung des Vorfalls bei Nulliparen. Surg. gynéc. a. obst.

20. Nov.. S. 534. Smith, Stephan, Reminiszenzen zweier Epochen von Anästhesie und Asepsis. Bull. Johns Hopkin. Hosp. XXX. S. 343.

142

Literatm·-Verzeiehnis.

Solms, Über zufällige Blasenverletzungen bei Scheidenoperationen. Dtsch.

med. Woch. H. XL S. 1279. Souper, Metastatische Staphylokokkeninfektion der Niere. Brit. m. J. 
1920. Nr. 3087. Steward, Klinisehe Vorlesung über die Behandlung der septischen Peritonitis. Brit, m. J. 20. April. Stracker, Ein schmerzverhindernder Kunstgriff bei der Lokalanästhesie. Zbl. f. Chir. 20. H. 9. Stroeder, Zur Trendelenburgschen Operation der Blasen-Scheidenfistel. Frauenarzt, 19. H. 10. Strohmayer, Zur Frage der künstlichen Sterilisierung der Frau aus eugenischer Indikation. Dtsch. med. Woch. 20. Nr. 14 u. 15. Síronndorf, Tracheloplastik bei chronischer Endocervicitis. Surg. gynéc.

a. obst. 20. Nov. S. 535. Szénás, Behandlung der weiblichen Gonorrhöe. Budapest! Orvosillisás. 19. Nr. 17. Walíher, Fibromyxoma lig. rotundi. Rév. mem. de gynéc. et d'obst. Febr. 19.

Wattsider, Übergangsformen gutartiger und bösartiger Vorgänge in Ovarien. Weinzierl, Seltene Koitusverletzung. Zbl. f. Gyn. 16. X. S. 1181. Wintz, Die physiologisch-chemische Wirkung des

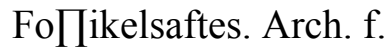

Gyn. Bd. 113. H. 3. S. 457. Zerleiel, Neues Verfahren bei Uterusinversion: Colpo-hystertomia ant.

mit Vaginofixation. Gynéc. et obstét. Nr. 2. S. 120. Zimmermann, Untersuchungen über die Häufigkeit des Auftretens von

Isoagglutinen und Isohämolysinen in Hinblick auf die Bluttransfusion.

Zbl. f. Gyn. 9. X. S. 1146. Zweífel, E., Wirkt fötales Serum artfremd auf das Muttertier ? Eine bio-

logische Studie zur Aufklärung der Eklampsie vom Standpunkt der

Anaphylaxieforschung. München u. Wiesbaden. 20. J. Bergmann.

Strahlentherapie.

Dietlen, Vorschlag zur Einschränkung der Gefahr von Röntgenschädigungen

und zur Gewinnung eines einheitlichen Dosierungsverfahrens. Munch.

med. Woch. 19. XL S. 1355. Drießen, Zur Verhütung filterloser Bestrahlung bei der

Tiefentherapie.

Zbl f. Gyn. 30. X. S. 1258. Fraenkel, M., Die Röntgenreizdosen in der Gynäkologie mit besonderer

Berücksichtigung der Karzinombekämpfung. Zbl. f. Gyn. 6. XL

S. 1288. Halberstaedter u. Tugendreich, Die Gefahr der Hautschädigungen bei der

Röntgendiagnostik. Berl. klin. Woch. 15. XL S. 1091. Lenk, Die Sensibilisierungsfrage in der

Strahlentherapie. Erwiderung auf

die gleichnamige Arbeit von Prof, von Rhorer in Nr. 39 d. W. Dtsch.

med. Woch. 4. XL 1255. Mansfeld, Über Metrasthenie, Tonisierung der Gebärmutter und

halbseitige

Rontgenkastration. Philips, Aufblähung des Colon als Beihilfe bei Röntgenuntersuchung.

Surg. Gynéc. obst. Nov. 20. S. 531. Zbl. f. Gyn. 3. X. S. 1260. Schanz, Luft- und

Lichtbehandlung. Strahlentherapie. IX. S. 2. S. 544. 